\title{
Research of variations of ionospheric parameters during the local meteorological phenomena in the Kaliningrad region
}

\author{
Olga Borchevkina $^{1, *}$, Ivan Karpov $^{1,2}$, Alexandra Ilminskaya ${ }^{1}$, Yuliya Kurdyaeva $^{1}$, Alexey \\ Karpov $^{1}$, and Nina Korenkova ${ }^{2}$ \\ ${ }^{1}$ Immanuel Kant Baltic Federal University, 236006 Kaliningrad, Russia \\ ${ }^{2}$ West department IZMIRAN, 236041 Kaliningrad, Russia
}

\begin{abstract}
The paper presents observations of atmospheric and ionospheric parameters during strong meteorological disturbances (storms) in the Kaliningrad region. The analysis of ionospheric observations has shown that during meteorological storms the amplitude of diurnal variations in TEC decreases to $50 \%$; and in foF 2 , to $15 \%$ as compared to quiet days. The revealed changes in ionospheric conditions during meteorological storms are regularly registered and represent a characteristic feature of the meteorological effect on the ionosphere. Modeling studies of the vertical propagation of AGW from the Earth's surface showed that such waves quickly (within $\sim 15 \mathrm{~min}$ ) reach altitudes of the upper atmosphere $(\sim 300 \mathrm{~km})$. The refraction and dissipation of waves in the upper atmosphere produces perturbations of the background state of the atmosphere and gives rise to the waveguide propagation of infrasonic wave components. The observed manifestations of TEC disturbances caused by AGW propagating from the lower atmosphere can be explained by the diurnal variation of the altitude of the ionosphere and the waveguide propagation of infrasonic waves.
\end{abstract}

\section{Introduction}

Meteorological processes are an important source of acoustic-gravity waves (AGW) in the atmosphere. Results of multiple experimental and theoretical studies suggest that different dynamic processes in the lower atmosphere and on Earth's surface, which are caused, for example, by meteorological, seismological, and other events, have a significant effect on ionospheric conditions. Assessments of the impact of such processes in the lower atmosphere on ionospheric conditions are reported in [1-4].

In particular, changes in the structure of ionospheric variations during meteorological disturbances are analyzed in [5-9]. Strong meteorological disturbances are characteristic of the climate of the Kaliningrad region. This allows us to identify meteorological effects in changes of ionospheric conditions.

\footnotetext{
*Corresponding author: opsuslova@gmail.com
} 


\section{The model of atmospheric processes}

The propagation of AGW in the lower atmosphere is theoretically studied using numerical models that take into account a variety of physical processes involved in the propagation of waves. The analysis of accumulation of commutation errors is studied in details in [10-12]. The constructed method allows the simulation of waves for times up to several tens of hours, and to distances up to several thousand kilometers and from the Earth's surface to the thermosphere heights without significant computational errors. This allows, in particular, computation of the impact of waves on the atmosphere and make simulation of wave propagation with consideration of changing of the medium properties due to transfer of energy of dissipated waves to the medium.

[13] explores methods of mathematical modeling the spread of AGW in the upper atmosphere from the surface source. According to theoretical studies, that above the source at an altitude of $\sim 200 \mathrm{~km}$ due to the dissipation of the AGW heating a region of the atmosphere with a horizontal scale of about $1000 \mathrm{~km}$ with a duration of operation of the power $\sim 1$ hour. The emergence of the heated region changes the propagation conditions of AGW and leads to the waveguide propagation of waves with periods shorter than the Brunt-Vaisala period. The upper boundary of the waveguide is formed heated region of the upper atmosphere. In the figure shows mean temperature in the upper atmosphere and wave temperature perturbations in the upper atmosphere produced by a ground-based AGW source. 2 hours after the power switching can be noted a significant change in wave propagation mode is manifested in the character of the waveguide propagation of atmospheric waves in the region below $\sim 200 \mathrm{~km}$, and the absence of wave disturbances above $250-300 \mathrm{~km}$.
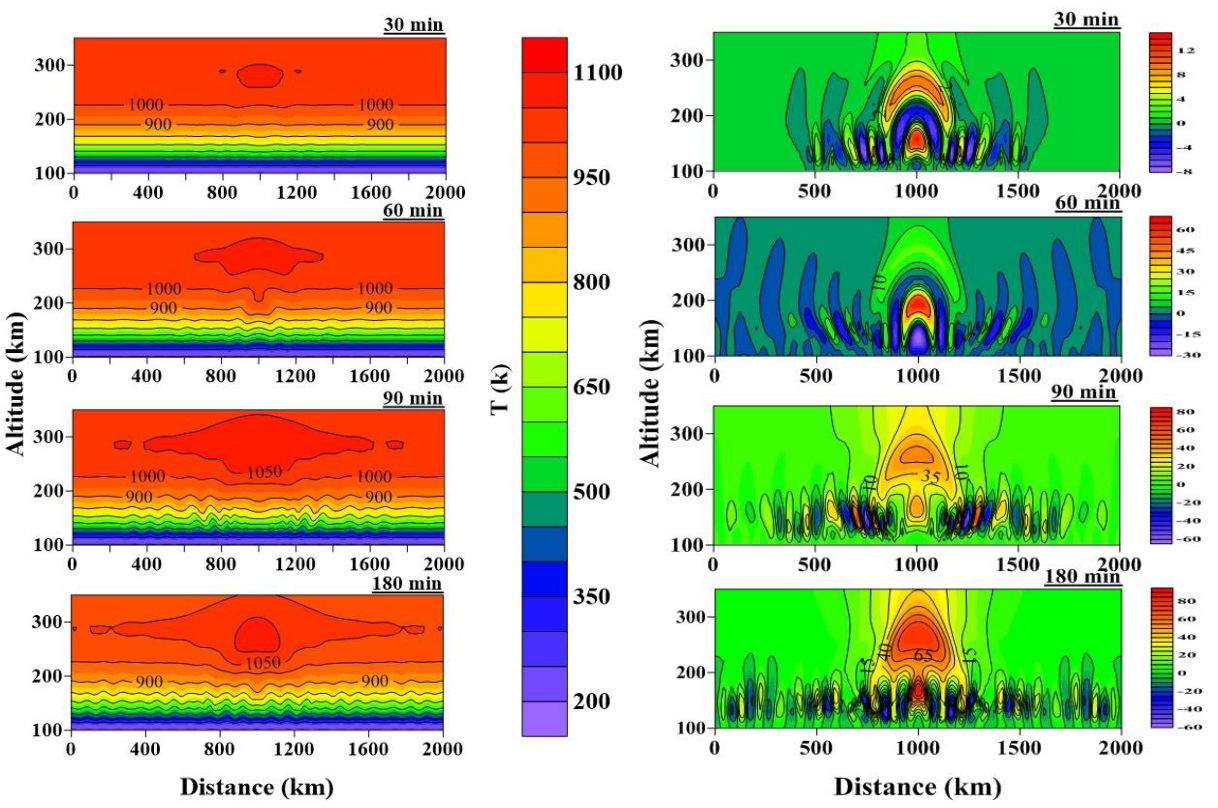

Fig. 1. Mean temperature in the upper atmosphere (left); 30, 60, 90 and 180 min. after start of operation of the ground-based AGW source. Wave temperature perturbations in the upper atmosphere produced by a ground-based AGW source (right) 30,60, 90 and $180 \mathrm{~min}$. after the start of operation of the ground-based AGW source. The horizontal coordinate of the source is $1000 \mathrm{~km}$;

\section{Diurnal variations in atmospheric and ionospheric parameters during a meteorological storm}


To examine ionospheric dynamics in conditions of meteorological disturbances, we have selected periods of the strongest meteorological storms in the Kaliningrad region. We analyze ionospheric data, using observations of the F2 layer critical frequency (foF2) and total electron content (TEC) obtained at the station Kaliningrad. Methods of determining foF2 from observations, made by the ionosonde Parus, and TEC by analyzing signals from GNSS satellites are described in $[14,15]$. Data on meteorological conditions have been taken from www.rp5.ru.

This paper focuses on rapidly growing meteorological disturbances followed by high winds of force $6-8$ on the Beaufort scale. Such storms in the Kaliningrad region generally last less than two days. To identify characteristic features of ionospheric disturbances arising during meteorological disturbances, we consider an observation period that began five days before a storm and ended five days after it. As weather parameters, we analyze diurnal variations in atmospheric pressure $\mathrm{P} 0$ at the altitude of the station and maximum gusts of wind at an altitude of $10 \mathrm{~m}$ over a 3-hour period between observations - the parameter FF3. To minimize the influence of geomagnetic factors on ionospheric variations, we chose meteorological disturbances under quiet geomagnetic conditions.

Figure 3 illustrates variations in atmospheric and ionospheric parameters during a meteorological disturbance of August 16, 2010. On August 13-23, geomagnetic conditions were quiet; $K_{\mathrm{p}} \leq 3$ (Figure 2 ). Solar activity changed very little, decreasing from $F 10.7=86$ on August 13 to $F 10.7=76$ on August 23.
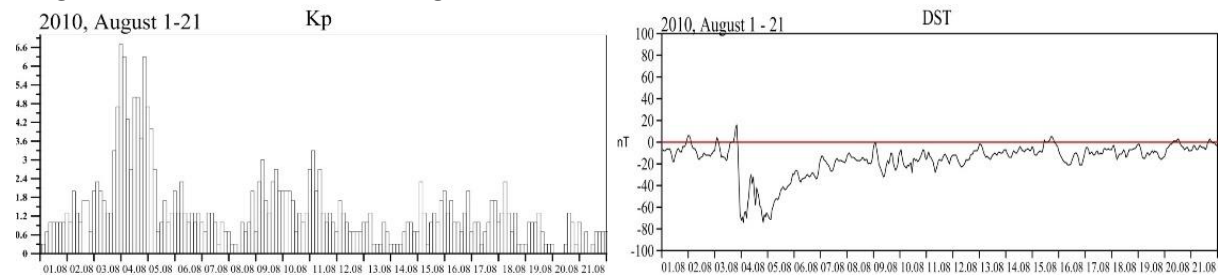

Fig. 2. Geomagnetic conditions in August 2010.

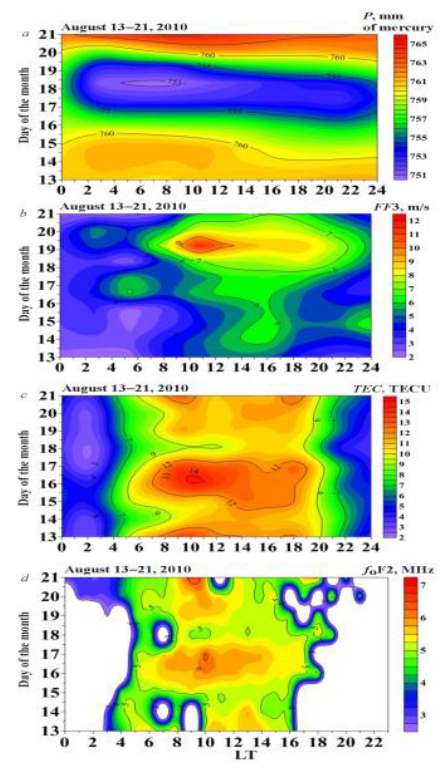

Fig. 3. Diurnal variations in surface air pressure (a); diurnal wind speed variations (b); diurnal TEC variations (c); diurnal foF2 variations (d) on August 13-21, 2010 according to observations from the station Kaliningrad. 
It is apparent that atmospheric pressure began to drop on August 14, reached its minimum on August 18, on the day of the storm, and began to rise on August 20 (Figure 3, a). The wind speed during the observation period increased largely during daytime on August 18-19, reaching its peak values of $\sim 14 \mathrm{~m} / \mathrm{s}$ on August 19. Figure $3 c, d$ shows that TEC and $f_{\mathrm{o}} \mathrm{F} 2$ disturbances were pronounced on August 14 and 18 (the white color in Figure 3, $d$ indicates observation areas in which we failed to find critical frequency values owing to strong absorption in the $\mathrm{E}$ and $\mathrm{D}$ regions). Those days, decreases in daytime TEC values run to $\sim 30-40 \%$ and in the critical frequency, to $\sim 15-20 \%$ as compared to stormless days.

Similarly, were analyzed another 15 meteorological storms [16]. In the study of some it was observed that during periods of meteorological disturbances occur in the ionosphere diffusivity and F scattering. As can be seen from the figure 4, while weather the storm on August 16 these phenomena have taken place mostly at night time.

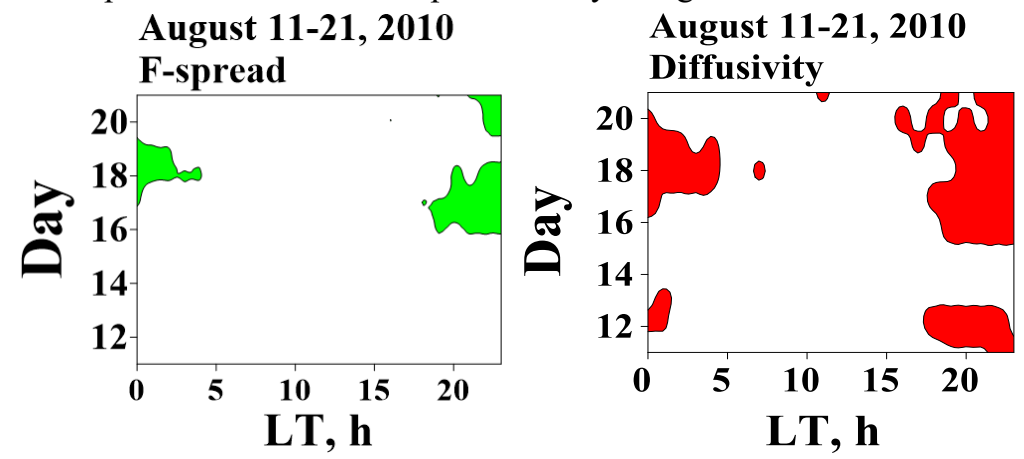

Fig. 4. Phenomena diffusivity and $\mathrm{F}$ scattering during periods of meteorological disturbances on August 13-21, 2010 according to observations from the station Kaliningrad.

\section{Discussion}

We can suppose that rapid changes in meteorological conditions during a storm favour excitation of AGWs over a wide range of periods. Propagation of AGWs to the upper atmosphere and their dissipation there result in the observed ionospheric disturbances. Theoretical studies of AGW propagation from sources in the lower atmosphere suggest that waves of this range can quickly reach the upper atmosphere and due to dissipation can give rise to large-scale disturbances, specifically to local heating regions. Noticeable large-scale disturbances in the upper atmosphere conditioned by dissipation of AGWs propagating from a source in the lower atmosphere are observed 1-2 hr after activation of the source [17]. A local heating region appearing in the upper atmosphere should affect the ionization balance in the ionosphere and lead to a decrease in the ionospheric electron density due to the growing influence of recombination processes.

Figure 5 shows the spectra of variations of the total electron content (TEC, an ionospheric parameter) with periods of 2 to $20 \mathrm{~min}$, changing during observations, over the KLG midlatitude station $\left(54.42^{\circ} \mathrm{N}, 20.27^{\circ} \mathrm{E}\right)$. Analysis of TEC results showed that during the period of meteorological storms intensified infrasonic components with periods of 6-10 minutes, lasting for days after the storm. 

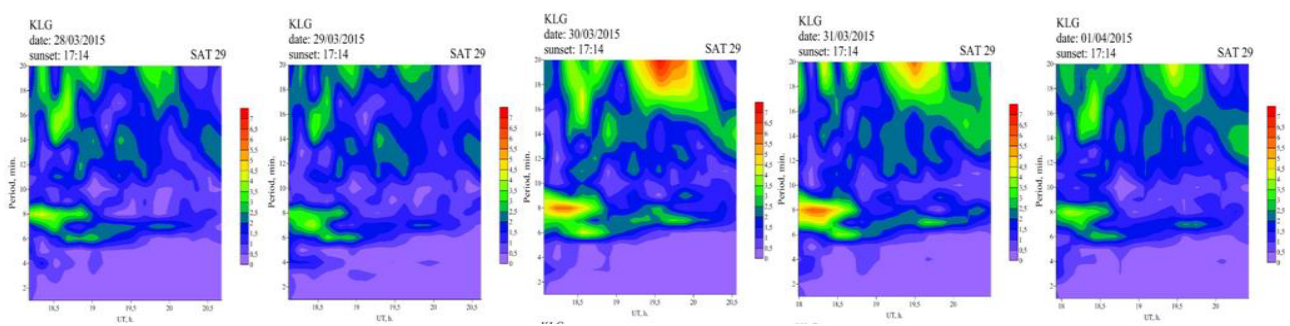

Fig. 5. Analysis of TEC results in signals from some GPS satellites during the period of meteorological storms March 29-30, 2015.

\section{Conclusion}

1. During the period of meteorological perturbations marked decreasing in the amplitude of diurnal variations of TEC up to $\sim 50 \%$; for foF $2-\sim 20 \%$. in relation to meteorologically calm days;

2. During the period of meteorological perturbations observed phenomena of Fscattering and diffuse in $\mathrm{F}$ region of the ionosphere;

3. Analysis of spectra of atmospheric variations detects the existence of harmonics with periods of acoustic gravity waves and infrasound waves in the ionosphere during meteorological storm;

4. AGWs, excited by localized sources on the Earth's surface, propagate to the heights of the upper atmosphere and ionosphere. The process of propagation of AGWs accompanied by interrelated effects due to the wave dissipation in the upper atmosphere: heating of the atmosphere by waves, which leads to local changes in atmospheric conditions, and waveguide propagation of wave perturbations with periods of less than a period Vaisala Brent in the disturbed atmosphere, at altitudes below $200 \mathrm{~km}$;

5. The most probable cause of ionospheric perturbations is the processes of formation of local area heating the thermosphere due to AGWs dissipation coming into the upper atmosphere from the area of meteorological perturbations in the lower atmosphere.

These investigations were performed with financial support of the Russian Foundation for Basic Research Grants No. 15-05-01665 and State task Education and Science Ministry of the Russian Federation, the competitive part of the task No 3.1127.2014/K.

\section{References}

1. D. Altadill, E.M. Apostolov, J.G. Solé, Ch. Jacobi, Physics and Chemistry of the Earth, Part C: Solar, Terrestrial \& Planetary Science 26, 6 (2001)

2. P. Sauli, J. Boska, Physics and Chemistry of the Earth, Part C: Solar, Terrestrial \& Planetary Science 26, 6 (2001)

3. J. Lastovicka, P. Sauli, Advances in Space Research. 24, 11 (1999)

4. D.C. Fritts, M.J. Alexander, Reviews of Geophysics 41, 1 (2003)

5. M.A. Chernigovskaya, B.G. Shpynev, K.G. Ratovsky, Journal of Atmospheric and SolarTerrestrial Physics 11, 22 (2015)

6. P. Sauli, J. Boska, Journal of Atmospheric and Solar-Terrestrial Physics 63, 9 (2001)

7. S.L. Vadas, H.-L. Liu. Journal of Geophysical Research: Space Physics 114, A10310 (2009) 
8. T. Sindelarova, D. Buresova, J. Chum, F. Hruska, Advances in Space Research 43, (2009)

9. A.S. Polyakova, N.P. Perevalova, Advances in Space Research (includes Cospar Information Bulletin) 52, 8 (2013)

10. S.P. Kshevetskii Analytycal and numerical investigation of nonlinear internal gravity waves. Nonlinear processess in geophysics 8, 37-51 (2001)

11. S. P. Kshevetskii, Computational mathematics and mathematical physics 41, 12 (2001)

12. S.P. Kshevetskii Computational Mathematics and Mathematical Physics 41, 2. (2001)

13. I.V. Karpov, S.P. Kshevetsky, O.P. Borchevkina, A.V. Radievsky, A.I. Karpov, Russian Journal of Physical Chemistry B 10, 1 (2016)

14. A.L. Karpenko, N.I. Manaenkova, Geologishe Rundshau 85, 1 (1996)

15. L.W. Baran, I.I. Shagimuratov, N.J. Tepenitzina The Use of GPS for Ionospheric Studies. Artificial satellites. Journal of Planetary Geodesy. 1997, vol. 32, no. 1, pp. 49-60; 16. I.V. Karpov, O.P. Borchevkina, R.Z. Dadashev, A.V. Ilminskaya, Solar-Terrestrial Physics 2, 2 (2016)

17. I. V. Karpov, S. P. Kshevetskii, Geomagnetism and Aeronomy 54, 4 (2014)

18. http://www.rp5.ru 